\title{
Laser-induced ultrafast demagnetization time and spin moment in ferromagnets: First-principles calculation
}

\author{
G. P. Zhang* \\ Department of Physics, Indiana State University, Terre Haute, IN 47809 \\ M. S. Si \\ Key Laboratory for Magnetism and Magnetic Materials of the Ministry of Education, \\ Lanzhou University, Lanzhou 730000, China \\ Thomas F. George \\ Office of the Chancellor and Center for Nanoscience \\ Departments of Chemistry 83 Biochemistry and Physics 83 Astronomy \\ University of Missouri-St. Louis, St. Louis, MO 63121
}

(Dated: March 3, 2022)

\begin{abstract}
When a laser pulse excites a ferromagnet, its spin undergoes a dramatic change. The initial demagnetization process is very fast. Experimentally, it is found that the demagnetization time is related to the spin moment in the sample. In this study, we employ the first-principles method to directly simulate such a process. We use the fixed spin moment method to change the spin moment in ferromagnetic nickel, and then we employ the Liouville equation to couple the laser pulse to the system. We find that in general the dependence of demagnetization time on the spin moment is nonlinear: It decreases with the spin moment up to a point, after which an increase with the spin moment is observed, followed by a second decrease. To understand this, we employ an extended Heisenberg model, which includes both the exchange interaction and spin-orbit coupling. The model directly links the demagnetization rate to the spin moment itself and demonstrates analytically that the spin relaxes more slowly with a small spin moment. A future experimental test of our predictions is needed.

PACS numbers: 75.78.Jp, 75.40.Gb, 78.20.Ls, 75.70.-i
\end{abstract}

Keywords: 
More than a decade ago, Beaurepaire et al. [1] demonstrated that a femtosecond laser pulse could demagnetize a nickel thin film in less than 1 picosecond, very attractive for future ultrafast magnetic storage devices. This inspired intensive experimental and theoretical investigations [2 14], with an even faster demagnetization reported [15, 16]. However, what determines the demagnetization time $\tau_{m}$ is a complex and challenging question, since multiple parameters, both intrinsic (material properties) and extrinsic (laser pulse), jointly play a role. With decades of experimental efforts, accurate demagnetization times are now available at least for ferromagnetic $3 d$ transition metals. Bcc Fe has a spin moment of $M=2.2 \mu_{\mathrm{B}}$ and demagnetization time of $\tau_{m}=98 \pm 26 \mathrm{fs}$ [17], and fcc Ni has a spin moment of $0.6 \mu_{\mathrm{B}}$ and $\tau_{m}=157 \pm 9 \mathrm{fs}$ [17] and 70-200 fs [18]. Therefore, it appears that $\tau_{m}$ is inversely proportional to $M$, but this trend is stopped at hcp Co. Its moment is $1.6 \mu_{\mathrm{B}}$, but $\tau_{m}=160-240 \mathrm{fs}$ [18], the longest among the three. This highlights the challenge and complicated nature of $\tau_{m}$, which is also affected by the exchange interaction. Since the demagnetization time is at the heart of the laser-induced ultrafast demagnetization process, a detailed investigation is very appropriate at this time.

In this paper, we focus on fcc Ni. We employ the density functional theory and the fixed spin moment method to gradually tune the spin moment, without strongly affecting other parameters. Then we carry out a dynamic simulation by solving the Liouville equation to compute the dynamic change of the density matrices at each $\mathbf{k}$ point, from which the spin moment change is computed. We can determine the demagnetization time for each spin moment. With an excitation by a 36 fs laser pulse, $\tau_{m}$ is found to decrease with the spin moment until $0.35 \mu_{\mathrm{B}}$, after which a small increase is observed, before the final decrease with the spin moment. The entire dependence appears highly nonlinear, but the general trend is clear: $\tau_{m}$ decreases with $M$. To explain this general trend, we adopt an extended Heisenberg model, which includes both the exchange interaction and spin-orbit coupling. We can analytically show that a larger spin moment indeed leads to a larger demagnetization rate, or shorter demagnetization time.

To systematically investigate the effect of the spin moment on the demagnetization time, we resort to the fixed spin moment method (FSM) [19]. FSM was originally designed to study the complex phase diagrams in complicated magnetic systems, where competing phases coexist on a similar energy scale. Within FSM, one starts from a normal spinpolarized DFT calculation and computes the spin moment. Then, one checks whether 
the spin moment matches the desired one. If not, one refills the spin-up and spin-down states to get the desired spin moment and forms a new charge density and potential for the next iteration until convergence. Different from the usual FSM, after the self-consistency is reached, we perform an extra run including the spin-orbit coupling, so we can investigate the spin moment change under laser excitation. We choose six spin moments, $0.19 \mu_{\mathrm{B}}, 0.27 \mu_{\mathrm{B}}$, $0.37 \mu_{\mathrm{B}}, 0.44 \mu_{\mathrm{B}}, 0.52 \mu_{\mathrm{B}}$ and $0.64 \mu_{\mathrm{B}}$. We use $104^{3} \mathrm{k}$ points to converge our results. Our real-time spin-moment change is computed first by solving the Liouville equation [20 22]

$$
i \hbar\left\langle n k\left|\frac{\partial \rho}{\partial t}\right| m k\right\rangle=\left\langle n k\left|\left[H_{0}+H_{I}, \rho\right]\right| m k\right\rangle,
$$

where $|m k\rangle$ is a band state $m$ at $k$. Once we obtain the density matrix, we trace over the product of the spin operator and density matrix to find the spin moment, $M_{z}=\operatorname{Tr}\left(\rho(t) S_{z}\right)$. Here $H_{0}$ is the Hamiltonian for the system, and $H_{I}$ is the interaction Hamiltonian between the laser field and the system,

$$
H_{I}=\sum_{k} \sum_{n m} \mathbf{E}(\mathrm{t}) \cdot \mathbf{D}_{k ; n m} \rho_{k ; n m}
$$

where $\mathbf{D}$ is the dipole operator. The laser field is

$$
\mathbf{E}(\mathrm{t})=\mathbf{A} \mathrm{e}^{-t^{2} / \tau^{2}} \cos (\omega t)
$$

where we align the laser polarization $\mathbf{A}$ along the $x$-axis, and the magnetization quantization axis is along the z-axis. Our laser duration $\tau$ is $36 \mathrm{fs}$, the photon energy is $\hbar \omega=2 \mathrm{eV}$, and the field amplitude $|\mathbf{A}|$ is $0.05 \mathrm{~V} / \AA$, which corresponds to a fluence of $11.5 \mathrm{~mJ} / \mathrm{cm}^{2}$, while experimentally the fluence ranges from $0.6 \mathrm{~mJ} / \mathrm{cm}^{2}$ [23] to $35 \mathrm{~mJ} / \mathrm{cm}^{2}$ [24]. Figure 11(a) shows the spin moment changes as a function of time for the spin moment of $0.37 \mu_{\mathrm{B}}$. We notice that the spin drops very quickly upon the laser excitation, since the electrons in metals are mobile and can be easily excited out of the Fermi sea, as there is no gap blocking such a process, as expected from the Fermi liquid theory. The time at the first spin minimum is defined as the demagnetization time $\tau_{m}$. For each spin moment, we carry out a similar calculation, and the detailed dependence on the spin moment is shown in Fig. 1(b). We find that the demagnetization time decreases very quickly with spin moment increase from 0.19 to $0.35 \mu_{\mathrm{B}}$. However, when the spin moment is above $0.35 \mu_{\mathrm{B}}$, it saturates, and interestingly, there is a small increase up to $0.45 \mu_{\mathrm{B}}$ before a further reduction with the spin moment. This dependence has never been reported before. 
We want to know whether the charge response already shows some important differences. Figure 2(a) shows the electric polarization as a function of time for the two spin moments of 0.19 (solid line) and 0.64 (long-dashed line) $\mu_{\mathrm{B}}$. It is interesting that the electric polarization behaves quite differently for these two cases. Since in the present cases our electric and laser polarizations are perpendicular to each other, the strong beating due to the laser field is strongly suppressed, in comparison to the collinear excitation [22]. The excitation for 0.19 $\mu_{\mathrm{B}}$ is much stronger than $0.64 \mu_{\mathrm{B}}$ and has prominent oscillations. For $0.64 \mu_{\mathrm{B}}$, the oscillation is weaker. There is a clear decoupling between the charge and spin response. Figure 2(b) shows that their respective spin changes are very different and always slower than the charge response. The net reduction of the spin change is larger for $0.64 \mu_{\mathrm{B}}$ than $0.19 \mu_{\mathrm{B}}$. This is because once the spin moment is small, there is no room for further reduction.

We can reveal some additional insights into the general trend through a model. For magnets, regardless of ferromagnets, antiferromagnets or ferrimagnets, a minimum model must include the exchange interaction. It is this interaction that sustains the long-range magnetic ordering across different lattice sites. However, including the exchange interaction is not enough to understand magnetization changes since the total spin momentum is a conserved quantity without spin-orbit coupling. We note in passing that in the traditional magnetism theory, the spin moment change is built in from the beginning through the BoseEinstein distribution of magnons, so that it does not apply here. With these considerations, we start from the spin-orbit-coupled Heisenberg model [25],

$$
H_{0}=-\sum_{i j} J \hat{\overrightarrow{s_{i}}} \cdot \hat{\vec{s}}_{j}+\sum_{i} \lambda \hat{\vec{l}}_{i} \cdot \hat{\vec{s}}_{i}
$$

where $J$ is the exchange interaction between nearest-neighbor atomic sites $i$ and $j, \lambda$ is the spin-orbit coupling, $\hat{\vec{s}}_{i}$ is the spin operator at site $i$, and $\hat{\vec{l}}_{i}$ is the orbital operator at site $i$. Within the Heisenberg picture, the $z$-component of the spin momentum at site $i$ evolves according to

$$
\dot{\hat{s}}_{i z}=\lambda\left(\hat{l}_{i x} \hat{s}_{i y}-\hat{l}_{i y} \hat{s}_{i x}\right)-\sum_{j} J\left(\hat{s}_{i y} \hat{s}_{j x}-\hat{s}_{i x} \hat{s}_{j y}\right) .
$$

The total spin momentum change $\dot{\hat{S}}_{z}$ is a sum over all the sites,

$$
\dot{\hat{S}}_{z}=\sum_{i} \dot{\hat{s}}_{i z}=\sum_{i} \lambda\left(\hat{l}_{i x} \hat{s}_{i y}-\hat{l}_{i y} \hat{s}_{i x}\right)
$$

where the exchange interaction term drops out. To reveal the role of the exchange coupling 
between different sites, we first integrate similar equations like Eq. (5) for $\hat{s}_{i x}$ and $\hat{s}_{i y}$, and then substitute them back into Eq. (6). The resultant equation (6), that is linear in $\lambda$, is

$$
\begin{aligned}
\dot{\hat{S}}_{z} & \approx \lambda \sum_{i}\left(\hat{l}_{i x} \hat{s}_{i y}(-\infty)-\hat{l}_{i y} \hat{s}_{i x}(-\infty)\right)-\lambda J \int_{-\infty}^{t} \sum_{i}\left(\hat{l}_{i x} \hat{s}_{i x}+\hat{l}_{i y} \hat{s}_{i y}\right) \hat{S}_{z} d t^{\prime} \\
& +\lambda J \int_{-\infty}^{t} \sum_{i} \hat{l}_{i x} \hat{s}_{i z} \hat{S}_{x} d t^{\prime}+\lambda J \int_{-\infty}^{t} \sum_{i} \hat{l}_{i y} \hat{s}_{i z} \hat{S}_{y} d t^{\prime}
\end{aligned}
$$

where $\hat{l}$ is a function of $t$, not $t^{\prime}$, which should not be integrated over. The first term on the right-hand side represents the contribution from the orbital momentum. The second term is directly proportional to the spin momentum $\hat{S}_{z}$ itself and represents the spin relaxation. The last two terms are the precessional terms since they are linked to the $x$ - and $y$-components of the spin momentum. Next, we assume initially that the spin is along the $z$-axis, so $\left\langle\hat{s}_{i x}(-\infty)\right\rangle=\left\langle\hat{s}_{i y}(-\infty)\right\rangle=0$. To first order in the spin-orbit basis [20], the last two terms are zero, so we also ignore them and only keep terms that contain $\hat{S}_{z}$. This approximation is crude, but in the beginning of spin dynamics, the demagnetization is dominant, and the spin precession is expected to be small. Under these approximations, we find

$$
\dot{\hat{S}}_{z} \approx-\frac{1}{2} \lambda J \int_{-\infty}^{t} \sum_{i}\left(\hat{l}_{i}^{\dagger} \hat{s}_{i}^{-}+\hat{l}_{i}^{-} \hat{s}_{i}^{\dagger}\right) \hat{S}_{z} d t^{\prime} .
$$

This is the master equation of the (de)magnetization process. For the first time, the spin momentum change rate is linked to the spin $s$ and orbital $l$ momenta, exchange interaction $J$ and spin-orbit coupling $\lambda$. The master equation shows that the spin momentum rate depends on the history of the spin momentum itself, a non-Markovian process. Note that both $s$ and $S$ affect the demagnetization rate. We see that for a larger $S_{z}$, its (de)magnetization rate $\dot{S}_{z}$ is larger. This means that for the same amount of spin moment change, it needs less time, or a shorter demagnetization time. This is consistent with our numerical calculation above. It is the direct coupling of the spin to the orbital degree of freedom that allows the spin to act upon itself self-consistently. The fact that the product of spin-orbit coupling and exchange interaction enters the rate equation highlights the critical role of the exchange interaction as the chief protector for magnetic ordering and spin-orbit coupling as the main channel for demagnetization. If $J$ is very small, we have Eq. (6) to determine the demagnetization time, which is normally very long, in particular in magnetic semiconductors. A strong $J$ shortens the demagnetization time, which matches the experimentally observed time scale. 
Because of this convoluted interaction, the spin moment affects the demagnetization time nonlinearly.

In conclusion, we have investigated how the demagnetization time depends on the spin moment in ferromagnetic fcc Ni. We employ the fixed spin moment method to systematically change the spin moment. For each spin moment, we compute the laser-induced ultrafast spin moment change as a function of time, from which we determine the demagnetization time. Our results show that in general the demagnetization time becomes shorter with a larger spin moment, and the dependence is highly nonlinear. Quantitatively, we find that $\tau_{m}$ decreases with $M_{z}$ precipitously up to $0.35 \mu_{\mathrm{B}}$ before a small increase around $0.45 \mu_{\mathrm{B}}$. A further decrease is observed afterward. To reveal some further insights, we adopt the extended Heisenberg model, which includes both the exchange interaction and spin-orbit coupling. We demonstrate that the generic feature is indeed reproduced. The reduction of the demagnetization time with the spin moment originates from the sharp increase of the demagnetization rate through the spin moment itself. The exchange interaction and spin-orbit coupling jointly determine the time scale of the demagnetization. The direct relation between the demagnetization time and exchange interaction is consistent with the experimental observation [17]. A future experimental test of our prediction is much needed, but is potentially challenging since other parameters may contribute. However, one may dope the system with some impurities [17], or employ the temperature [26], laser fluence [18], or pressure [27]. Since the demagnetization time is an integrated part of femtomagnetism, we believe that our finding will pave the way to reveal the intricate mechanism of laser-induced ultrafast demagnetization.

This work was solely supported by the U.S. Department of Energy under Contract No. DE-FG02-06ER46304. Part of the work was done on Indiana State University's Quantum cluster and High performance computers. This research used resources of the National Energy Research Scientific Computing Center, which is supported by the Office of Science of the U.S. Department of Energy under Contract No. DE-AC02-05CH11231. Our calculations also used resources of the Argonne Leadership Computing Facility at Argonne National Laboratory, which is supported by the Office of Science of the U.S. Department of Energy under Contract No. DE-AC02-06CH11357.

*gpzhang@indstate.edu 
[1] E. Beaurepaire, J. -C. Merle, A. Daunois, and J.-Y. Bigot, Phys. Rev. Lett. 76, 4250 (1996).

[2] G. P. Zhang, W. Hübner, E. Beaurepaire, and J.-Y. Bigot, Topics Appl. Phys. 83, 245 (2002).

[3] A. Kirilyuk, A. V. Kimel and Th. Rasing, Rev. Mod. Phys. 82, 2731 (2010).

[4] W. Hübner and G. P. Zhang, Phys. Rev. B 58, R5920 (1998).

[5] G. P. Zhang and W. Hübner, Phys. Rev. Lett. 85, 3025 (2000).

[6] G. Lefkidis, G. P. Zhang, and W. Hübner, Phys. Rev. Lett. 103, 217401 (2009).

[7] D. Steiauf and M. Fähnle Phys. Rev. B 79, 140401 (2009); M. Fähnle and C. Illg, J. Phys.: Condens. Matter 23, 493201 (2011).

[8] J.-Y Bigot, M. Vomir and E. Beaurepaire, Nature Phys. 5, 515 (2009).

[9] C. D. Stanciu, F. Hansteen, A. V. Kimel, A. Kirilyuk, A. Tsukamoto, A. Itoh, and Th. Rasing, Phys. Rev. Lett. 99, 047601 (2007).

[10] G. Lefkidis and W. Hübner, J. Mag. Mag. Mater. 321, 979 (2009).

[11] R. Carley, K. Dobrich, B. Frietsch, C. Gahl, M. Teichmann, O. Schwarzkopf, P. Wernet, and M. Weinelt, Phys. Rev. Lett. 109, 057401 (2012).

[12] M. Battiato, K. Carva, and P. M. Oppeneer, Phys. Rev. B 86, 024404 (2012).

[13] K. Vahaplar, A. M. Kalashnikova, A. V. Kimel, D. Hinzke, U. Nowak, R. Chantrell, A. Tsukamoto, A. Itoh, A. Kirilyuk, and Th. Rasing, Phys. Rev. Lett. 103, 117201 (2009).

[14] H.-S. Rhie, H. A. Durr, and W. Eberhardt, Phys. Rev. Lett. 90, 247201 (2003).

[15] C. La-O-Vorakiat et al., Phys. Rev. X 2, 011005 (2012).

[16] M. Krauß, T. Roth, S. Alebrand, D. Steil, M. Cinchetti, M. Aeschlimann, and H. C. Schneider, Phys. Rev. B 80, 180407(R) (2009).

[17] S. Mathias et al., PNAS 109, 4792 (2012).

[18] B. Koopmans et al., Nat. Mater. 9, 259 (2010).

[19] K. Schwarz and P. Mohn, J. Phys. F: Met. Phys. 14, L129 (1984); V. L. Moruzzi, Phys. Rev. Lett. 57, 2211 (1986); A. R. Williams, V. L. Moruzzi, J. Kübler, and K. Schwarz, Bull. Am. Phys. Soc. 29, 278 (1984); P. H. Dederichs, S. Blügel, R. Zoller, and H. Akai, Phys. Rev. Lett. 53, 2512 (1984).

[20] G. P. Zhang, Y. Bai, and T. F. George, Phys. Rev. B 80, 214415 (2009).

[21] G. P. Zhang, W. Hübner, G. Lefkidis, Y. Bai, and T. F. George, Nature Phys. 5, 499 (2009). 

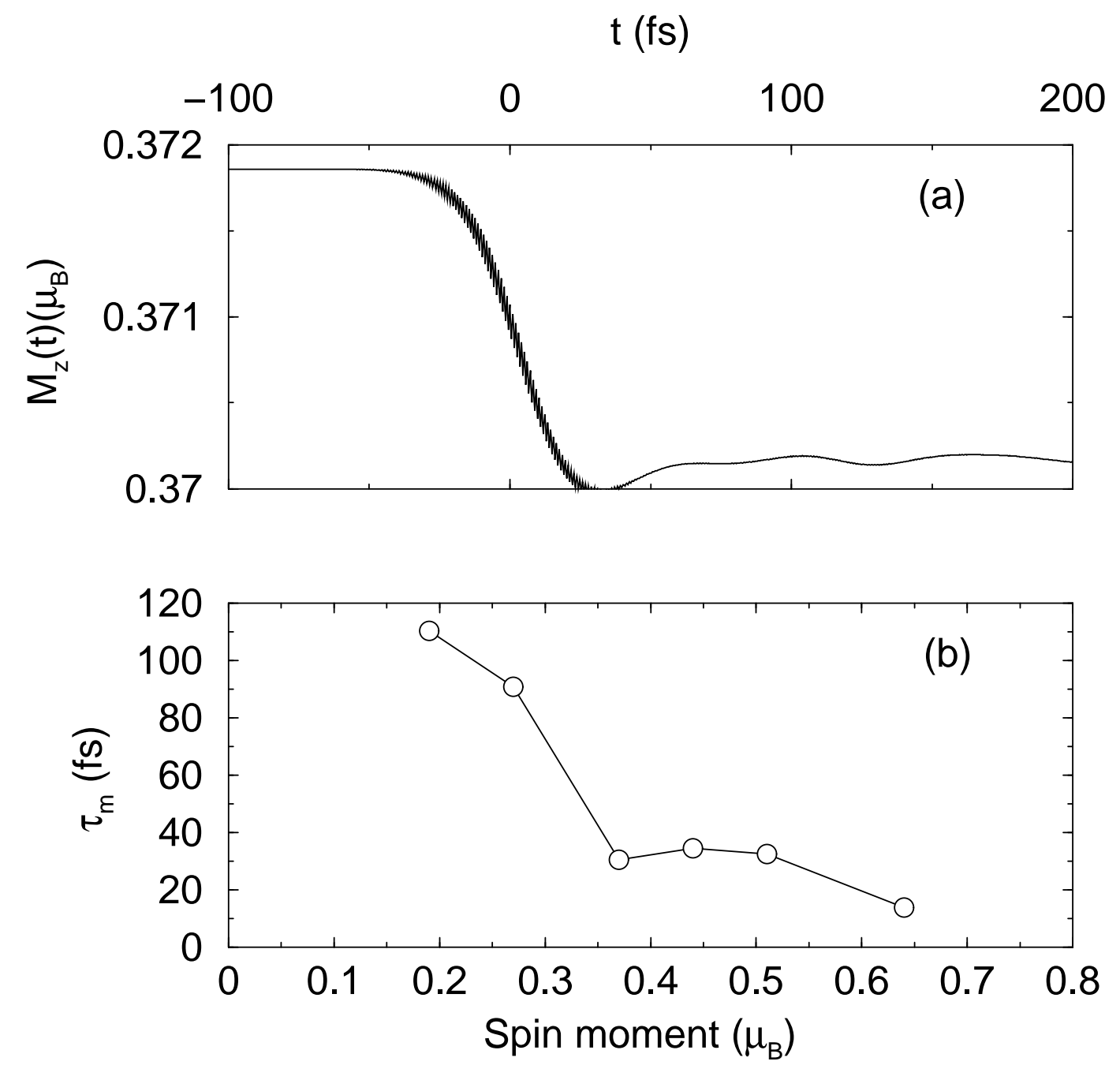

FIG. 1: (a) Laser-induced ultrafast demagnetization, where the spin moment is $0.37 \mu_{\mathrm{B}}$. (b) Demagnetization time $\tau_{m}$ as a function of spin moment. We choose six different spin moments.

[22] G. P. Zhang, Y. Bai, W. Hübner, G. Lefkidis, and T. F. George, J. Appl. Phys. 103, 07B113 (2008).

[23] B. Koopmans et al., J. Appl. Phys. 87, 5070 (2000).

[24] D. Cheskis et al., Phys. Rev. B 72, 014437 (2005).

[25] G. P. Zhang, J. Phys.: Condens. Matter 23, 206005 (2011).

[26] B. Koopmans, Topics in Appl. Phys. 87, 253 (2003); B. Koopmans, M. van Kampen and W. J. de Jonge, J. Phys. Condens. Matter 15, S723 (2003).

[27] R. Torchio, Y. O. Kvashnin, S. Pascarelli, O. Mathon, C. Marini, L. Genovese, P. Bruno, G. Garbarino, A. Dewaele, F. Occelli, and P. Loubeyre, Phys. Rev. Lett. 107, 237202 (2011). 


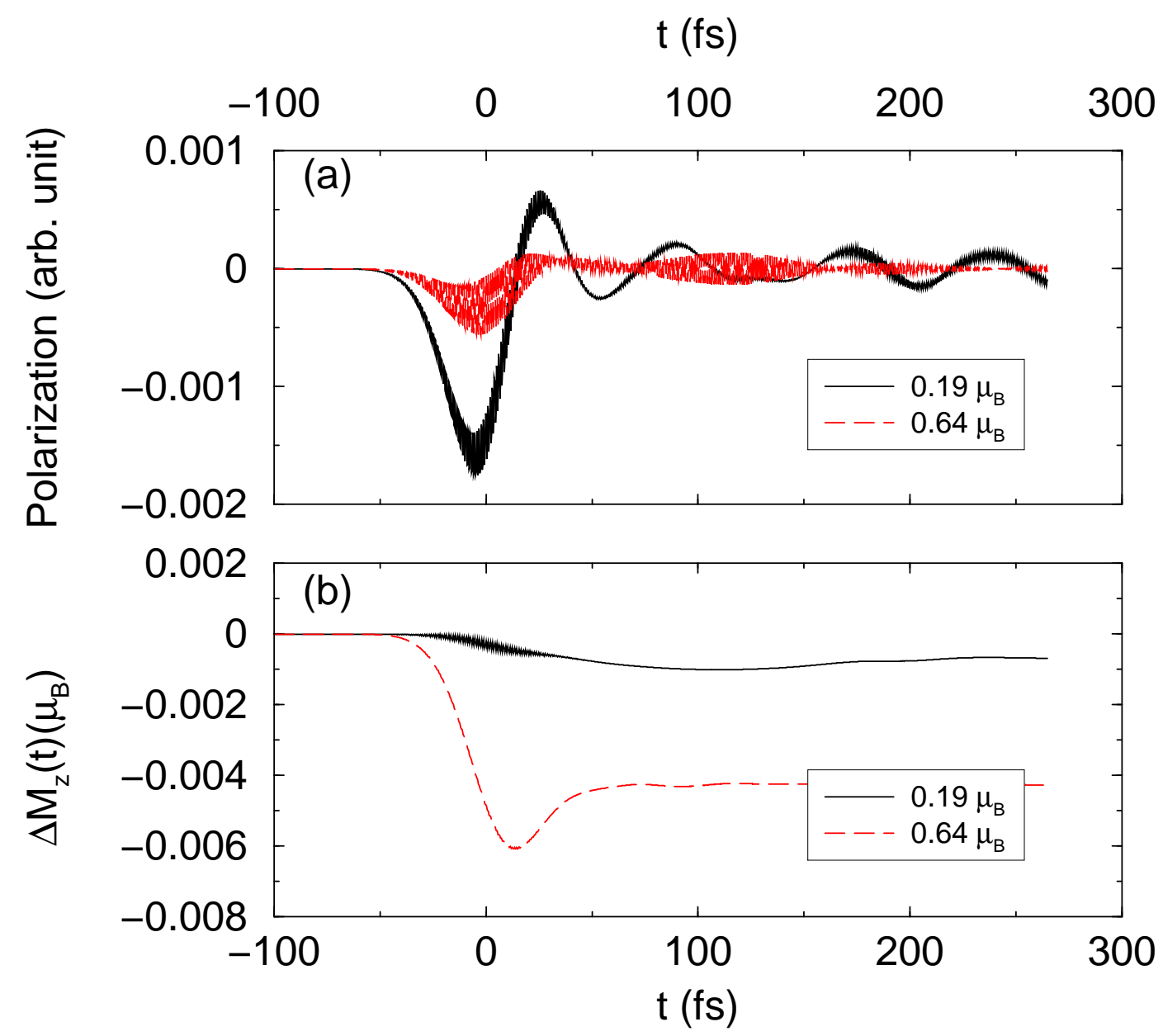

FIG. 2: (a) Electric polarization change as a function of time for two spin moments at 0.19 (solid line) and $0.64 \mu_{\mathrm{B}}$ (long dashed line). (b) Spin moment change $\Delta M(t)$ as a function of time $t$. The notation of the lines is same as (a). 